Research Article

\title{
Stochastic Air-Fuel Ratio Control of Compressed Natural Gas Engines Using State Observer
}

\author{
Jun Yang $\mathbb{D}^{D}$, Jian Wang $\mathbb{D}^{\mathrm{D}}$, Xuesheng Zhou, and Yanxiao Li \\ Department of Automotive Engineering, Shandong Jiaotong University, Jinan 250357, China \\ Correspondence should be addressed to Jun Yang; yang222401@163.com
}

Received 1 December 2019; Revised 31 January 2020; Accepted 10 February 2020; Published 12 March 2020

Academic Editor: Vincenzo Bianco

Copyright (c) 2020 Jun Yang et al. This is an open access article distributed under the Creative Commons Attribution License, which permits unrestricted use, distribution, and reproduction in any medium, provided the original work is properly cited.

In this paper, the air-fuel ratio regulation problem of compressed natural gas (CNG) engines considering stochastic $L_{2}$ disturbance attenuation is researched. A state observer is designed to overcome the unmeasurability of the total air mass and total fuel mass in the cylinder, since the residual air and residual fuel that are included in the residual gas are unmeasured and the residual gas reflects stochasticity. With the proposed state observer, a stochastic robust air-fuel ratio regulator is proposed by using a CNG engine dynamic model to attenuate the uncertain cyclic fluctuation of the fresh air, and the augmented closed-loop system is mean-square stable. A validation of the proposed stochastic robust air-fuel ratio regulator is carried out by the numerical simulation of two working conditions. The accuracy control of the air-fuel ratio is realized by the proposed stochastic robust airfuel ratio regulator, which in turn leads to an improvement in fuel economy and emission performance of the CNG engines.

\section{Introduction}

Natural gas is an acknowledged appropriate optional fuel, since it is characterized by clean combustion, a preferable fuel economy, richness, and low cost among various alternative fuels. Consequently, researchers have paid much attention to natural gas engines to achieve fuel economy and low emissions.

A gasoline engine with the multipoint injection system was modified to a CNG engine by [1], from which the results of the experiment had been developed. To calculate the airfuel ratio in the chamber of precombustion, a model reflecting various fuel flow rates and mixtures was established in [2]. The emission and combustion performance affected by compression ratio of natural gas engines with excess hydrogen in the case of various air-fuel ratios was investigated in [3] through experiments. The results on the performance of the emission and combustion of natural gas engines, which were affected by the composition of natural gas, were reviewed in [4]. The emissions of particles were researched in [5] for a natural gas engine, and experiments were conducted with a natural gas engine that was modified from a gasoline-fuelled engine. The cyclic variation in the combustion in natural gas engines with premixed and lean burn characteristics was discussed in [6]. Experimental research on improving the thermal efficiency by enhancing the compression ratio was carried out in [7], to achieve a greater ratio of expansion. Integrated effects of injection timing and boost pressure on combustion and performance of a direct injection CNG engine were researched in [8], and the experimental results demonstrated that an improvement was achieved when the boost pressures were larger than $7.5 \mathrm{Kpa}$ at every operating point. To discover the potential application of a CNG engine to transport, the technology of a lean mixture ignited by a laser was investigated in [9], considering various excess air ratios and compression ratios. Current research results on the efficiency, emission, and combustion performances of CNG engines were reviewed in [10]. A summary of the injection technologies to improve gas engine performance was given in [11], and the injection timing and compression ratio were assessed to be the major factors affecting the combustion and performance of CNG engines. Experimental results on the emission characteristics and performance of a CNG engine that was modified from a port injection SI engine were presented in [12], and the results showed that despite the low-power and high energy 
consumption, the emissions were improved dramatically. A adaptive technology for the fuel-air ratio regulation of CNG engines was researched in [13] to attenuate the uncertain variation in the methane content. The combustion and emission performance of a stratified air-fuel mixture was investigated for a CNG engine in [14], and an enhancement of the overall efficiency of the engine was realized compared to the premixed case.

However, it is known that, the fuel economy and emissions of CNG engines are heavily affected by the control accuracy of the in-cylinder air-fuel ratio, which is defined as the ratio of the total air mass to the total fuel mass in the cylinder. Unfortunately, the masses of the total air and the total fuel in the cylinder are unmeasured, since the so-called residual gas trapped in the cylinder at the end of the exhaust stroke contains the residual air and residual fuel, which are unmeasured, and the cyclic variation of the residual gas has stochasticity. Moreover, the fresh air mass exhibits an uncertain cyclic fluctuation and also affects the control accuracy of the in-cylinder air-fuel ratio. In this paper, a state observer-based stochastic robust air-fuel ratio regulator is presented to overcome the errors in the estimation of the masses of the total air and the total fuel, and the fluctuation of the fresh air mass, which prominently influence the accuracy of the air-fuel ratio control of CNG engines. Consequently, by using the designed stochastic robust air-fuel ratio regulator, the fuel economy and emission performance of the CNG engines will be enhanced. The regulator is designed based on the dynamic model of $\mathrm{CNG}$ engines which represents the cyclic transient characteristics of the air path and the fuel path. A numerical simulation is given to demonstrate the performance of the designed stochastic robust air-fuel ratio regulator.

\section{Control Problem Formulation}

The exchange process of the in-cylinder gas in CNG engines is depicted in Figure 1.

Since CNG engines have similar dynamics to gasoline engines, a dynamic model with discrete-time characteristics is given with the assumption that the mixture flowing to the manifold during the pressure equalization process breaths into the cylinder in the intake stroke [15]:

$$
\begin{aligned}
& M_{a}(k+1)=\left(M_{a}(k)-\lambda_{d} \mu M_{f}(k)\right) r(k)+M_{a n}(k), \\
& M_{f}(k+1)=M_{f}(k)(1-\mu) r(k)+M_{f n}(k),
\end{aligned}
$$

where $M_{a}(k)$ denotes the total mass of the in-cylinder air, $M_{f}(k)$ denotes the total mass of the in-cylinder fuel, $\lambda_{d}$ denotes the ideal air-fuel ratio, $\mu \in(0,1)$ denotes the combustion efficiency, $r(k)$ denotes the fraction of the residual gas, $M_{a n}(k)$ denotes the mass of the intake air, and $M_{f n}(k)$ denotes the mass of the intake fuel. The regulation error of the air-fuel ratio $y(k)$ is defined as

$$
\begin{aligned}
y(k) & =M_{a}(k)-\lambda_{d} M_{f}(k), \\
M_{a n}(k) & =\Delta M_{a n}(k)+M_{a n 0},
\end{aligned}
$$

where the cyclic fluctuation of the intake air $\Delta M_{a n}(k)$ is modeled as a disturbance pertaining to $L_{2}\lceil 0, \infty\rceil$, and $M_{a n 0}$ denotes the constant part of the intake air mass $M_{a n}(k)$. From (1), (2), and (3), we have

$$
y(k+1)=r(k) y(k)+\Delta M_{a n}(k)-\lambda_{d} \Delta M_{f n}(k),
$$

where the fresh fuel cyclic variation $\Delta M_{f n}(k)$ is defined as

$$
\Delta M_{f n}(k)=M_{f n}(k)-M_{f n 0},
$$

and $M_{f n 0}$ denotes the constant part of the intake fuel mass $M_{f n}(k)$ and can be calculated by

$$
M_{f n 0}=\frac{M_{a n 0}}{\lambda_{d}},
$$

$r(k)$ is modeled as a Markov chain with discrete-time characteristics, and the prediction model of $r(k)$ in one step is shown as

$$
\widehat{r}(k+1)=\sum_{j=1}^{N} s_{j} p_{i j},
$$

where $\hat{r}(k+1)$ denotes the output of the model, $s_{j}$ denotes the state, and $p_{i j}$ denotes the transition probability in one step between $s_{i}$ and $s_{j}$. For system (4), we design a state observer and a feedback regulator:

$$
\begin{aligned}
\hat{y}(k+1) & =f\left(\hat{y}(k), \Delta M_{f n}(k), \lambda(k), \hat{\lambda}(k)\right), \\
M_{f n}(k) & =g\left(\hat{y}(k), M_{a n 0}, \lambda_{d}, r(k)\right),
\end{aligned}
$$

such that the following inequality,

$$
E \sum_{k=0}^{\infty} y^{2}(k)<\gamma \sum_{k=0}^{\infty} \Delta M_{a n}^{2}(k)
$$

holds for all $\Delta M_{\text {an }}^{2}(k)$. With the stochastic robust air-fuel ratio regulator (9), the augmented system that consists of (4) and (8) is mean-square stable.

2.1. Stochastic Robust Air-Fuel Ratio Controller. The design process of the observer-based stochastic robust air-fuel ratio regulator is described in this part. For the design of the observer, approximate linearization is used as follows:

$$
\begin{aligned}
\lambda(k)= & \frac{M_{a}(k)}{M_{f}(k)} \approx \frac{M_{a n 0}}{M_{f n 0}}+\frac{M_{a}(k)-M_{a n 0}}{M_{f n 0}} \\
& -\frac{M_{a n 0}\left(M_{f}(k)-M_{f n 0}\right)}{M_{f n 0}^{2}},
\end{aligned}
$$

since the air-fuel ratio is a nonlinear function of the total air mass and total fuel mass in the cylinder. Substituting (6) into (11), we have

$$
\lambda(k)-\lambda_{d} \approx \frac{M_{a}(k)}{M_{f n 0}}-\frac{M_{a n 0} M_{f}(k)}{M_{f n 0}^{2}}=\frac{y(k)}{M_{f n 0}},
$$

from which we can obtain that the air-fuel ratio is a linear function of $y(k)$. 


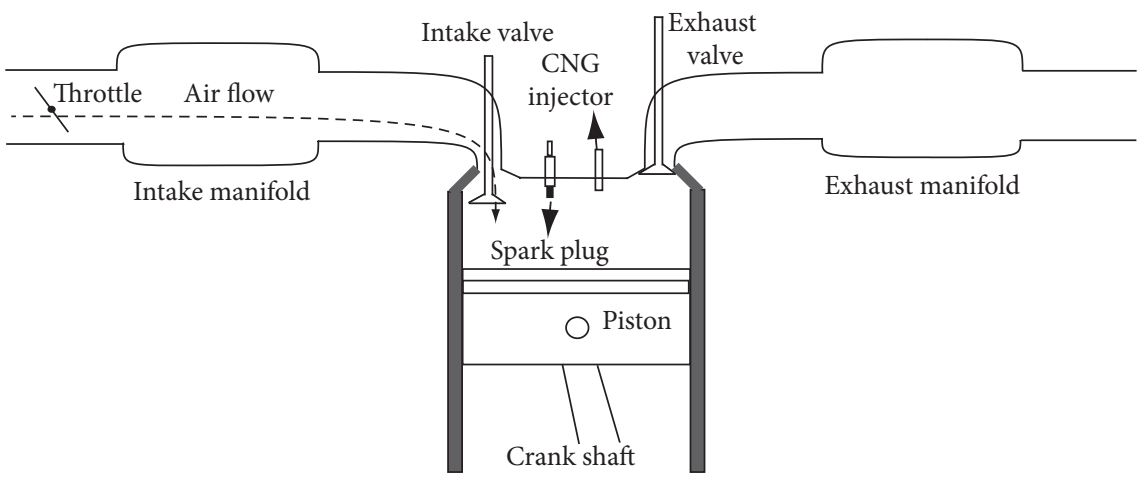

FIgURE 1: Schematic of the gas exchange system of the CNG engines.

Theorem 1. For system (4), setting a constant $\gamma>0$, if a set $\left\{\chi\left(s_{j}\right)>0, s_{i} \in \Omega\right\}$ exists, satisfying

$$
\gamma-\frac{2 \sum_{j=1}^{N} p_{i j} \chi\left(s_{j}\right) M_{f n 0}^{2}}{2 \sum_{j=1}^{N} p_{i j} \chi\left(s_{j}\right) G^{2}-\chi\left(s_{i}\right) M_{f n 0}^{2}+M_{f n 0}^{2} \Phi}>0,
$$

where

$$
\begin{gathered}
2 \sum_{j=1}^{N} p_{i j} \chi\left(s_{j}\right) G^{2}-\chi\left(s_{i}\right) M_{f n 0}^{2}+M_{f n 0}^{2} \Phi<0, \\
\Phi=\frac{\chi^{2}\left(s_{i}\right) M_{f n 0}^{2}}{2 \chi\left(s_{j}\right) M_{f n 0}^{2}-2 \sum_{j=1}^{N} p_{i j} \chi\left(s_{j}\right)\left(s_{j} M_{f n 0}-G\right)^{2}}>0,
\end{gathered}
$$

and $G$ is a design parameter, then there exist state observer and stochastic robust regulator:

$$
\begin{aligned}
\hat{y}(k+1) & =r(k) \hat{y}(k)-\lambda_{d} \Delta M_{f n}(k)+G(\lambda(k)-\hat{\lambda}(k)), \\
\Delta M_{f n}(k) & =\left(\frac{s_{i}}{\lambda_{d}}-\frac{G}{\lambda_{d} M_{f n 0}}\right) \hat{y}(k),
\end{aligned}
$$

such that system (4) exhibits $L_{2}$-gain performance $\gamma$ over $[0, \infty]$.

Proof. Select the stochastic Lyapunov function as

$$
V\left(k, r(k)=s_{i}\right)=\chi\left(s_{i}\right) \widehat{y}^{2}(k)+\chi\left(s_{i}\right) \varepsilon^{2}(k),
$$

where $\varepsilon$ is the observer error of the regulation error of the air-fuel ratio $y(k)$ and is defined as

$$
\varepsilon(k)=y(k)-\hat{y}(k) .
$$

Substituting (4), (12), and (16) into (19), we can obtain

$$
\begin{aligned}
& \varepsilon(k+1)=y(k+1)-\hat{y}(k+1) \\
& =r(k) y(k)+\Delta M_{a n}(k)-\lambda_{d} \Delta M_{f n}(k) \\
& -r(k) \hat{y}(k)+\lambda_{d} \Delta M_{f n}(k) \\
& -G(\lambda(k)-\hat{\lambda}(k))=r(k) \varepsilon(k)-\frac{G}{M_{f n 0}} \varepsilon(k)+\Delta M_{a n}(k) .
\end{aligned}
$$

From (16), (18), and (20), we have

$$
\begin{aligned}
\Delta V\left(k, r(k)=s_{i}\right)= & E\left[V(k+1, r(k+1)) \mid r(k)=s_{i}\right]-V\left(k, r(k)=s_{i}\right) \\
= & \sum_{j=1}^{N} p_{i j} \chi\left(s_{j}\right) \widehat{y}^{2}(k+1)-\chi\left(s_{i}\right) \widehat{y}^{2}(k)+\sum_{j=1}^{N} p_{i j} \chi\left(s_{j}\right) \varepsilon^{2}(k+1)-\chi\left(s_{i}\right) \varepsilon^{2}(k) \\
= & \sum_{j=1}^{N} p_{i j} \chi\left(s_{j}\right)\left(s_{i} \widehat{y}(k)-\lambda_{d} \Delta M_{f n}(k)+\frac{G}{M_{f n 0}} y(k)-\frac{G}{M_{f n 0}} \hat{y}(k)\right)^{2}-\chi\left(s_{i}\right) \widehat{y}^{2}(k) \\
& +\sum_{j=1}^{N} p_{i j} \chi\left(s_{j}\right)\left(s_{i} \varepsilon(k)-\frac{G}{M_{f n 0}} \varepsilon(k)+\Delta M_{a n}(k)\right)^{2}-\chi\left(s_{i}\right) \varepsilon^{2}(k) .
\end{aligned}
$$


Rearrange (21) as follows:

$$
\begin{aligned}
& \Delta V\left(k, r(k)=s_{j}\right) \\
& \leq 2 \sum_{j=1}^{N} p_{i j} \chi\left(s_{j}\right)\left(\left(s_{i}-\frac{G}{M_{f n 0}}\right) \hat{y}(k)-\lambda_{d} \Delta M_{f n}(k)\right)^{2} \\
& +2 \sum_{j=1}^{N} p_{i j} \chi\left(s_{j}\right) \frac{G^{2}}{M_{f n 0}^{2}} y^{2}(k)-\chi\left(s_{i}\right)(y(k)-\varepsilon(k))^{2} \\
& +2 \sum_{j=1}^{N} p_{i j} \chi\left(s_{j}\right)\left(s_{i} \varepsilon(k)-\frac{G}{M_{f n 0}} \varepsilon(k)\right)^{2}+2 \sum_{j=1}^{N} p_{i j} \chi\left(s_{j}\right) \Delta M_{a n}^{2}(k)-\chi\left(s_{i}\right) \varepsilon^{2}(k) \\
& \leq 2 \sum_{j=1}^{N} p_{i j} \chi\left(s_{j}\right)\left(\left(s_{i}-\frac{G}{M_{f n 0}}\right) \hat{y}(k)-\lambda_{d} \Delta M_{f n}(k)\right)^{2} \\
& -\frac{\chi\left(s_{i}\right) M_{f n 0}^{2}-2 \sum_{j=1}^{N} p_{i j} \chi\left(s_{j}\right) G^{2}-M_{f n 0}^{2} \Phi}{M_{f n 0}^{2}} y^{2}(k)-\Phi y^{2}(k) \\
& +2 \chi\left(s_{i}\right) y(k) \varepsilon(k)-\frac{\chi^{2}\left(s_{i}\right)}{\Phi} \varepsilon^{2}(k) \\
& +\frac{\chi\left(s_{i}\right) M_{f n 0}^{2}-2 \sum_{j=1}^{N} p_{i j} \chi\left(s_{j}\right) G^{2}-M_{f n 0}^{2} \Phi}{M_{f n 0}^{2}} \gamma \Delta M_{a n}^{2}(k) \\
& -\left(\frac{\chi\left(s_{i}\right) M_{f n 0}^{2}-2 \sum_{j=1}^{N} p_{i j} \chi\left(s_{j}\right) G^{2}-M_{f n 0}^{2}}{M_{f n 0}^{2}} \gamma-2 \sum_{j=1}^{N} p_{i j} \chi\left(s_{j}\right)\right) \Delta M_{a n}^{2}(k) .
\end{aligned}
$$

Based on (13)-(15) and (17), we obtain

$$
\Delta V\left(k, r(k)=s_{i}\right) \leq-\frac{\chi\left(s_{i}\right) M_{f n 0}^{2}-2 \sum_{j=1}^{N} p_{i j} \chi\left(s_{j}\right) G^{2}-M_{f n 0}^{2} \Phi}{M_{f n 0}^{2}} y^{2}(k)+\frac{\chi\left(s_{i}\right) M_{f n 0}^{2}-2 \sum_{j=1}^{N} p_{i j} \chi\left(s_{j}\right) G^{2}-M_{f n 0}^{2} \Phi}{M_{f n 0}^{2}} \gamma \Delta M_{a n}^{2}(k) .
$$

For every $s_{i},(23)$ is suitable, then,

$$
\begin{aligned}
& E V(k+1, r(k+1))-E V(k, r(k)) \\
& \quad=\sum_{i=1}^{N}\left(E\left[V(k+1, r(k+1)) \mid r(k)=s_{i}\right]-V\left(k, s_{i}\right)\right) p_{s_{i}} \\
& \quad \leq \sum_{j=1}^{N}\left(-\frac{\chi\left(s_{i}\right) M_{f n 0}^{2}-2 \sum_{j=1}^{N} p_{i j} \chi\left(s_{j}\right) G^{2}-M_{f n 0}^{2} \Phi}{M_{f n 0}^{2}} y^{2}(k)+\frac{\chi\left(s_{i}\right) M_{f n 0}^{2}-2 \sum_{j=1}^{N} p_{i j} \chi\left(s_{j}\right) G^{2}-M_{f n 0}^{2} \Phi}{M_{f n 0}^{2}} \gamma \Delta M_{a n}^{2}(k)\right) p_{s_{i}} \\
& \quad=-D E y^{2}(k)+\gamma D \Delta M_{a n}^{2}(k),
\end{aligned}
$$

where 


$$
D=\sum_{j=1}^{N}\left(\frac{\chi\left(s_{i}\right) M_{f n 0}^{2}-2 \sum_{j=1}^{N} p_{i j} \chi\left(s_{j}\right) G^{2}-M_{f n 0}^{2} \Phi}{M_{f n 0}^{2}}\right) p_{s_{i}}>0 .
$$

Summing both sides from 0 to $\infty$, we get

$$
\begin{aligned}
& \mathrm{EV}(\infty, r(\infty))-\mathrm{EV}(0, r(0)) \leq-\mathrm{DE} \sum_{k=0}^{\infty} y^{2}(k) \\
& \quad+\gamma D \sum_{k=0}^{\infty} \Delta M_{a n}^{2}(k) .
\end{aligned}
$$

Hence,

$$
E \sum_{k=0}^{\infty} y^{2}(k) \leq \frac{V(0, r(0))}{D}+\gamma \sum_{k=0}^{\infty} \Delta M_{a n}^{2}(k) .
$$

Therefore, system (4) exhibits $L_{2}$-gain performance $\gamma$ over $[0, \infty]$.

2.2. Stability Analysis. A stability analysis of the augmented closed-loop system of (4), (16), and (17) is presented. The definition of mean-square stable and the corresponding stability theorem that will be used in the design process is given in the following:

$$
\begin{aligned}
x(k+1) & =A(\xi(k)) x(k)+B(\xi(k)) \omega(k), \\
x(0) & =x_{0}, r(0)=r_{0},
\end{aligned}
$$

where $x(k)$ denotes the state of the system, $\xi(k)$ denotes a Markov chain with discrete-time characteristics, $\omega(k)$ denotes second-order Gaussian white noise with $E(\omega(k))=$ $\omega_{0}$ and $E\left(\omega(k) \omega^{T}(k)\right)=\Omega$, and $\omega^{T}(k)$ is the transpose of $\omega(k)$.

Definition 1 (see [16]). System (28) is said to be meansquare stable, if for every initial value $x(0)$ and initial distribution $\xi(0)$, the constants $q$ and $Q$ which are independent of $x(0)$ exist, so that

$$
\begin{gathered}
\|q(k)-q\| \longrightarrow 0, \quad \text { as } k \longrightarrow \infty, \\
\|Q(k)-Q\| \longrightarrow 0, \quad \text { as } k \longrightarrow \infty,
\end{gathered}
$$

where

$$
\begin{aligned}
q(k) & =\sum_{i=1}^{N} E\left(x(k) I_{\xi(k)=s_{i}}\right), \\
Q(k) & =\sum_{i=1}^{N} E\left(x(k) x^{T}(k) I_{\xi(k)=s_{i}}\right),
\end{aligned}
$$

and $I_{\{\}\}}$is the Dirac measure.

Lemma 1 (see [16]). If for every set of symmetric matrices $\left\{W\left(s_{i}\right)>0, i=1, \ldots, N\right\}$, a set of appropriate dimension matrices $\left\{\chi\left(s_{i}\right)>0, i=1, \ldots, N\right\}$ exists, so that

$$
\sum_{j=1}^{N} p_{i j} A^{T}\left(s_{i}\right) \chi\left(s_{j}\right) A\left(s_{i}\right)-\chi\left(s_{i}\right)=-W\left(s_{i}\right),
$$

where $A\left(s_{i}\right)$ means $\xi(k)=s_{i}$ in matrix $A$, and then system (28) is mean-square stable.

Theorem 2. For every initial value $(y(0), \hat{y}(0))^{T}$ and fluctuation $\Delta M_{a n}(k)$, the augmented closed-loop system of (4), (16), and (17) is mean-square stable.

Proof. From (4), (16), and (17), we can obtain

$$
\left(\begin{array}{c}
y(k+1) \\
\hat{y}(k+1)
\end{array}\right)=\left(\begin{array}{c}
r(k) r(k)-\frac{G}{M_{f n 0}} \\
\frac{G}{M_{f n 0}} \\
0
\end{array}\right)\left(\begin{array}{c}
y(k) \\
\hat{y}(k)
\end{array}\right)+\left(\begin{array}{c}
\Delta M_{a n}(k) \\
0
\end{array}\right) .
$$

Defining

$$
A=\left(\begin{array}{cc}
r(k) & r(k)-\frac{G}{M_{f n 0}} \\
\frac{G}{M_{f n 0}} & 0
\end{array}\right),
$$

and choosing

$$
\chi\left(s_{i}\right)=\chi=\left(\begin{array}{cc}
\frac{1}{\lambda_{d}} & 0 \\
0 & \lambda_{d}
\end{array}\right), \quad i=1, \ldots, N,
$$

we have

$$
\begin{gathered}
\sum_{j=1}^{N} p_{i j} A^{T}\left(s_{i}\right) \chi A\left(s_{i}\right)-\chi=-W\left(s_{i}\right), \\
W\left(s_{i}\right)=\left(\begin{array}{ll}
W_{11}\left(s_{i}\right) & W_{12}\left(s_{i}\right) \\
W_{21}\left(s_{i}\right) & W_{22}\left(s_{i}\right)
\end{array}\right),
\end{gathered}
$$

where

$$
\begin{aligned}
W_{11}\left(s_{i}\right) & =\frac{1}{\lambda_{d}}-\frac{s_{i}^{2}}{\lambda_{d}}-\frac{G^{2}}{\lambda_{d}^{2} M_{f n 0}^{2}}, \\
W_{12} & =-\frac{s_{i}}{\lambda_{d}}\left(s_{i}-\frac{G}{M_{f n 0}}\right), \\
W_{21} & =-\frac{s_{i}}{\lambda_{d}}\left(s_{i}-\frac{G}{M_{f n 0}}\right), \\
W_{22} & =\lambda_{d}-\frac{1}{\lambda_{d}}\left(s_{i}-\frac{G}{M_{f n 0}}\right)^{2} .
\end{aligned}
$$

From (14) and (15) and noting that $r(k) \in(0,0.5)$ [10], we obtain 


$$
\begin{array}{r}
\frac{1}{\lambda_{d}}-\frac{s_{i}^{2}}{\lambda_{d}}-\frac{G^{2}}{\lambda_{d}^{2} M_{f n 0}^{2}}>0, \\
\operatorname{det}\left(W\left(s_{i}\right)\right)>0,
\end{array}
$$

i.e., $W$ is a matrix with symmetric positive-definite characteristics. Therefore, the augmented system of (4), (16), and (17) is mean-square stable.

2.3. Simulation Verification. A numerical simulation of CNG engines is established by (1) and (2) and mean-value models [17-19] as follows:

$$
\begin{aligned}
y(k) & =M_{a}(k)-M_{f}(k) \lambda_{d}, \\
M_{a}(k+1) & =\left(M_{a}(k)-\lambda_{d} \mu M_{f}(k)\right) r(k)+M_{a n}(k), \\
M_{f}(k+1) & =M_{f}(k)(1-\mu) r(k)+M_{f n}(k), \\
\dot{M}_{a n} & =\frac{\rho_{a} V_{d} \eta_{v}}{4 \pi P_{a}} \omega_{e} P_{m}, \\
T_{e} & =\frac{H_{u} V_{d} \eta_{i} \eta_{v} P_{m}}{4 \pi R T_{m} \lambda}, \\
J \dot{\omega}_{e} & =T_{e}-T_{l}, \\
\dot{P}_{m} & =\frac{R T_{m}}{V_{m}}\left(\dot{M}_{i}-\dot{M}_{a n}\right), \\
\dot{M}_{i} & =s_{0}(1-\cos \phi) \frac{P_{a}}{\sqrt{R T_{a}}} \psi\left(\frac{P_{a}}{P_{m}}\right)
\end{aligned}
$$

where $T_{m}$ is $298.15 \mathrm{~K}, V_{m}$ is $5.897 \mathrm{E}-03 \mathrm{~m}^{3}, V_{d}$ is $5.76 E-04 \mathrm{~m}^{3}, s_{0}$ is $3.5 E-03 \mathrm{~m}^{2}, T_{a}$ is $298.15 \mathrm{~K}, \lambda_{d}$ is 17.4 , and

$$
\psi(s)= \begin{cases}s^{2 / k}\left(\frac{2 k}{k-1}(1-s)\right)^{k-1 / k}, & \text { if } s \geq\left(\frac{2}{k+1}\right)^{k / k-1}, \\ k\left(\frac{2}{k+1}\right)^{(k+1 / k-1)}, & \text { if otherwise. }\end{cases}
$$

The numerical simulation is conducted under working conditions $W_{1}$ and $W_{2}$. For working condition $W_{1}$, the engine revolution is $1200 \mathrm{rpm}$ and the external load is $60 \mathrm{Nm}$. For working condition $W_{2}$, the engine revolution is $1600 \mathrm{rpm}$ and the external load is $90 \mathrm{Nm}$. The measurement of the residual gas fraction of each cycle $r(k)$ is shown with the assumptions that the exhaust stroke is an adiabatic polytropic process, and the ideal gas constant of the exhaust stroke is unvaried [15]:

$$
r(k)=\frac{1}{\varepsilon}\left(\frac{P_{\mathrm{TDC}_{e}}(k)}{P_{\mathrm{BDC}_{e}}(k)}\right)^{1 / n},
$$

where $\varepsilon$ denotes the ratio of effective compression, $n$ denotes the polytropic value, $P_{\mathrm{TDC}_{e}}(k)$ denotes the cylinder pressure at the end of the exhaust stroke, and $P_{\mathrm{BDC}_{e}}(k)$ denotes the cylinder pressures at the end of the combustion stroke.
The samples of the residual gas fraction under $W_{1}$ and $W_{2}$ are separated into four segments, and the state spaces are given by calculating the average values of each segment, which are shown in the following equations:

$$
\begin{aligned}
& S_{W_{1}}=\{0.085,0.082,0.080,0.076\}, \\
& S_{W_{2}}=\{0.087,0.081,0.075,0.072\} .
\end{aligned}
$$

The probability matrices of the transition in one-step (46) and (47) are obtained by computing the value of the one-step transition frequency:

$$
\begin{gathered}
P_{W_{1}}=\left(\begin{array}{llll}
0.10 & 0.33 & 0.39 & 0.27 \\
0.11 & 0.14 & 0.39 & 0.36 \\
0.31 & 0.14 & 0.14 & 0.41 \\
0.41 & 0.31 & 0.10 & 0.18
\end{array}\right), \\
P_{W_{2}}=\left(\begin{array}{llll}
0.25 & 0.02 & 0.19 & 0.54 \\
0.51 & 0.24 & 0.04 & 0.21 \\
0.21 & 0.52 & 0.23 & 0.04 \\
0.03 & 0.21 & 0.55 & 0.21
\end{array}\right) .
\end{gathered}
$$

Based on (44)-(47), the prediction model in one-step of the residual gas fraction for the two working conditions is obtained. The parameter $G$ is set to $4.55 \times 10^{-7}, \gamma$ is set to 6.2 , and $\chi\left(s_{i}\right)$ is set to 1 for all of the working conditions. Figures 2 and 3 show the performances of the air-fuel ratio for $W_{1}$ and $W_{2}$, where $U_{\mathrm{SOR}}(k)$ denotes the proposed stochastic observer-based robust air-fuel ratio regulator and $U_{\text {open }}(k)$ denotes the open-loop regulator which is given as follows:

$$
U_{\text {open }}(k)=\frac{M_{a n 0}}{\lambda_{d}} .
$$

From Figures 2 and 3, we can observe that both $U_{\text {SOR }}(k)$ and $U_{\text {open }}(k)$ regulate the air-fuel ratio into the neighborhood of the ideal value 17.4, and the range of the air-fuel ratio fluctuation of $U_{\text {open }}(k)$ is larger than that of $U_{\text {SOR }}(k)$ under $W_{1}$ and $W_{2}$. In contrast, the range of the fresh fuel mass fluctuation of $U_{\text {open }}(k)$ is smaller than that of $U_{\text {SOR }}(k)$ under $W_{1}$ and $W_{2}$, which is found in Figures 4 and 5, since $U_{\text {open }}(k)$ does not focus on the errors of the estimation of the total air and the total fuel and the fluctuation of the fresh air mass. The performances of the observer values of $W_{1}$ and $W_{2}$ are exhibited in Figures 6 and 7 , which show that the observed values of the regulation error of the air-fuel ratio can be regulated into neighborhoods of the corresponding steady value. The size of the steady value is determined by the parameter $D$, which is defined by (25). Indeed, the range of the air-fuel ratio of typical CNG engines is between 10 to 22 [1], and the CNG engines are stable in this range. Therefore, the stable operation of the CNG engines in this paper can be ensured, since the range of the air-fuel ratio variation shown in Figures 2 and 3 is only 17 to 17.8 .

Table 1 shows the control performance over 20000 cycles of $U_{\mathrm{SOR}}(k)$ and $U_{\text {open }}(k)$. From Table 1 , we find that robustness is achieved by $U_{\mathrm{SOR}}(k)$ in the presence of a 


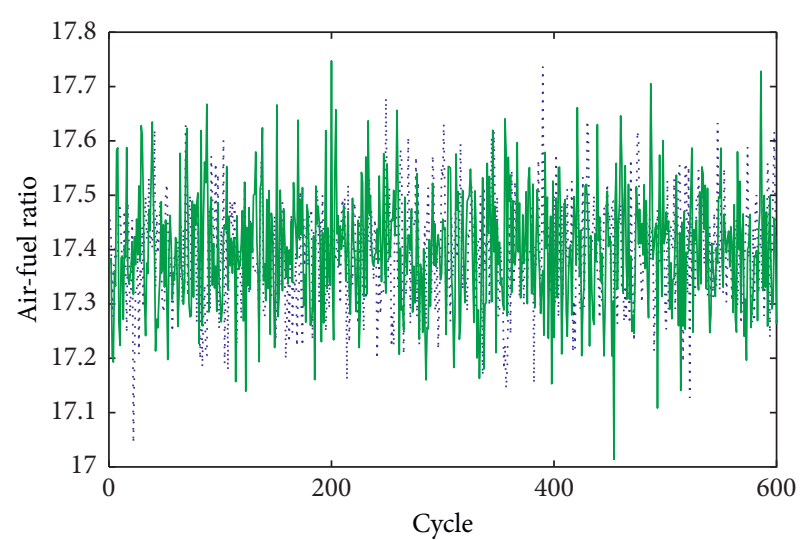

$$
\begin{aligned}
& U_{\mathrm{SOR}}(\mathrm{k}) \\
& -U_{\text {open }}(\mathrm{k})
\end{aligned}
$$

Figure 2: Air-fuel ratio of $W_{1}$.

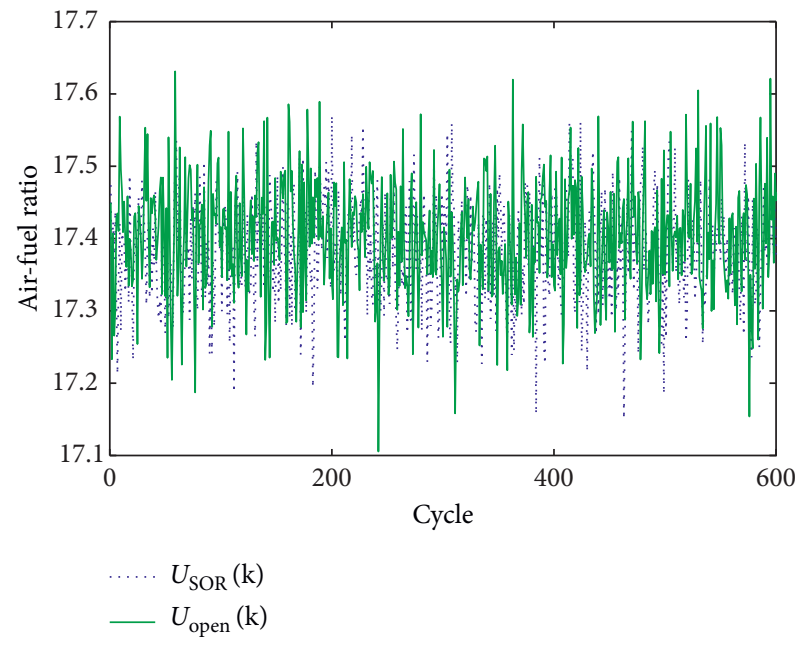

Figure 3: Air-fuel ratio of $W_{2}$.

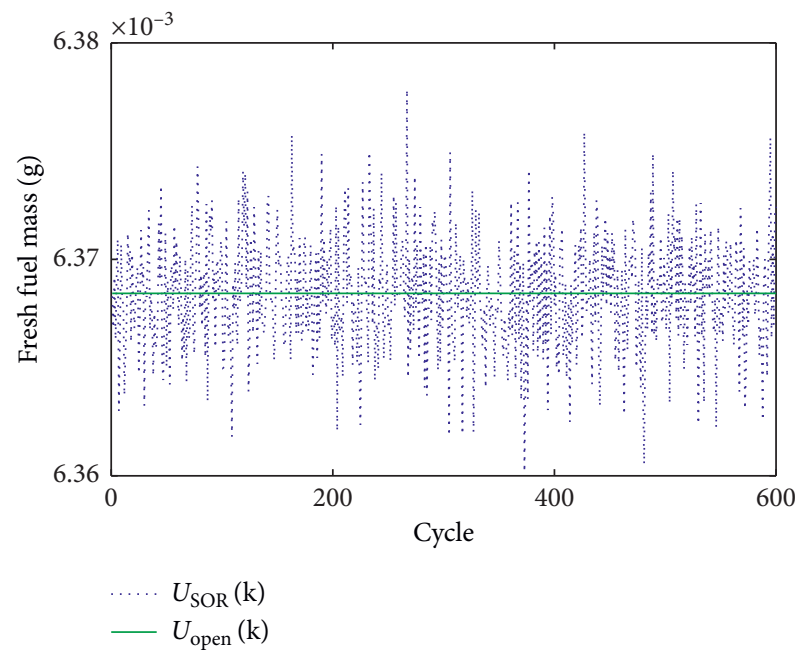

Figure 4: Fresh fuel mass of $W_{1}$.

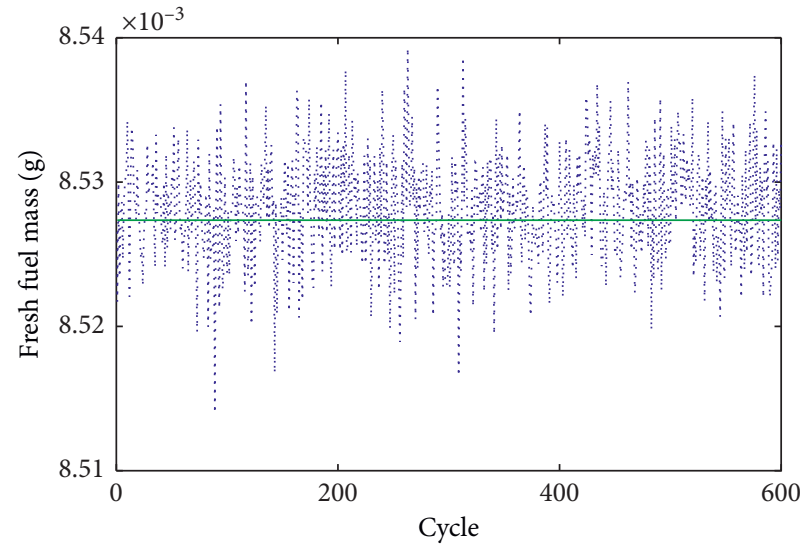

$\cdots \cdot U_{\mathrm{SOR}}(\mathrm{k})$
$-U_{\text {open }}(\mathrm{k})$

Figure 5: Fresh fuel mass of $W_{2}$.

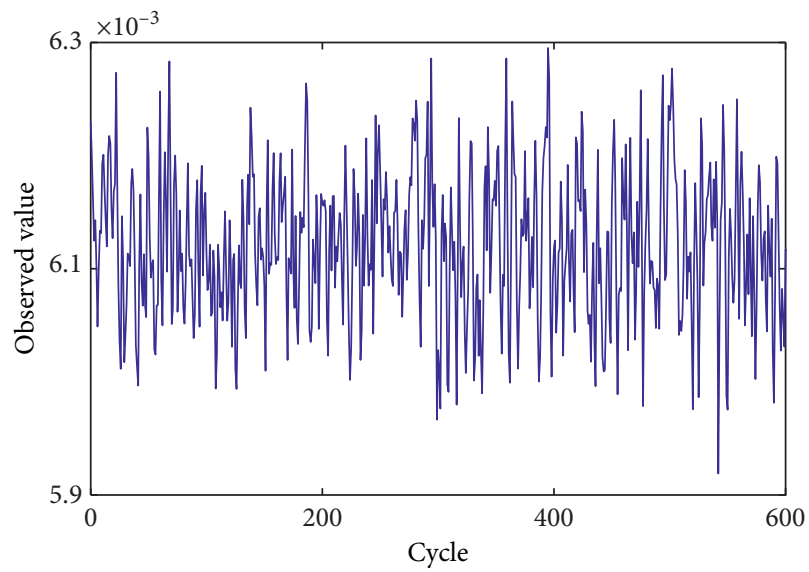

Figure 6: Observer value of $W_{1}$.

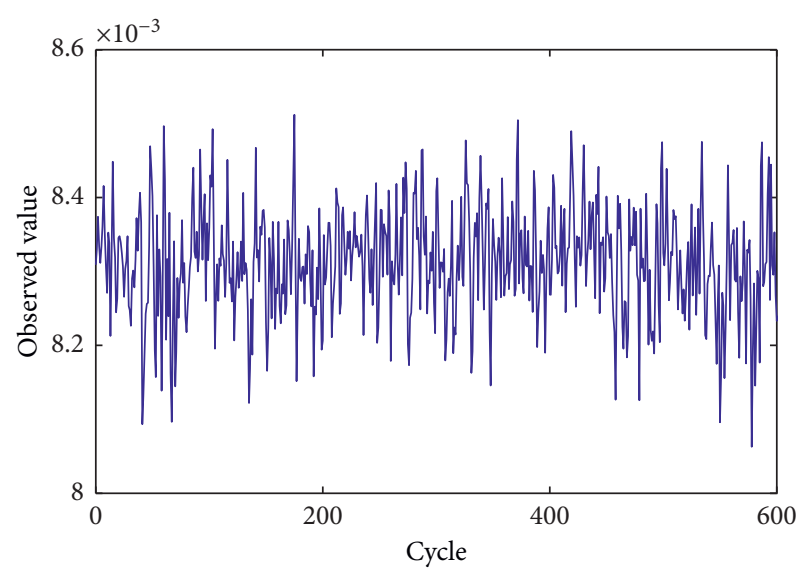

Figure 7: Observer value of $W_{2}$. 
TABLE 1: Control performance.

\begin{tabular}{lccc}
\hline & $J_{U_{\text {SOR }}(k)}(20000)$ & $J_{U_{\text {open }}(k)}(20000)$ & $\gamma$ \\
\hline$W_{1}$ & 0.8666 & 0.9993 & 6.2 \\
$W_{2}$ & 0.9144 & 1.0092 & 6.2 \\
\hline
\end{tabular}

fluctuation of the fresh air mass and $U_{\mathrm{SOR}}(k)$ achieves better performance than that of $U_{\text {open }}(k)$, where

$$
J(N)=\frac{\sum_{k=1}^{N} y^{T}(k) y(k)}{\sum_{k=1}^{N} \Delta M_{a n}^{T}(k) \Delta M_{a n}(k)} .
$$

\section{Conclusions}

This paper addressed the problem of state observer-based stochastic robust air-fuel ratio regulation of CNG engines, since the existence of errors in estimation of the masses of the total air and the total fuel, and the uncertain cyclic fluctuation of the fresh air mass heavily affect the control accuracy of the air-fuel ratio. A stochastic robust air-fuel ratio regulator has been designed based on approximate linearization, and the augmented closed-loop system achieves mean-square stable. A validation of the proposed stochastic robust air-fuel ratio regulator has been verified through a numerical simulation under two working conditions. Based on the implementation of the proposed stochastic robust air-fuel ratio regulator, the control accuracy of the air-fuel ratio has been improved and then the effect of the control inaccuracy of the air-fuel ratio on the fuel economy and emission performance of the CNG engines is attenuated. Considering alternative fuels, the control problem of gas engines fuelled by biogas will be our future work.

\section{Data Availability}

No data were used to support this study.

\section{Conflicts of Interest}

The authors declare that there are no conflicts of interest regarding the publication of this paper.

\section{Acknowledgments}

This work was supported by the National Natural Science Foundation (NNSF) of China under Grant 61803231.

\section{References}

[1] M. A. Kalam and H. H. Masjuki, "An experimental investigation of high performance natural gas engine with direct injection," Energy, vol. 36, no. 5, pp. 3563-3571, 2011.

[2] M. D. Ruter, D. B. Olsen, M. V. Scotto, and M. A. Perna, " $\mathrm{NO}_{X}$ reduction from a large bore natural gas engine via reformed natural gas prechamber fueling optimization," Fuel, vol. 91, no. 1, pp. 298-306, 2012.

[3] J. Zhao, F. Ma, X. Xiong et al., "Effects of compression ratio on the combustion and emission of a hydrogen enriched natural gas engine under different excess air ratio," Energy, vol. 59, pp. 658-665, 2013.

[4] A.-H. Kakaee, A. Paykani, and M. Ghajar, "The influence of fuel composition on the combustion and emission characteristics of natural gas fueled engines," Renewable and Sustainable Energy Reviews, vol. 38, pp. 64-78, 2014.

[5] J. Alanen, E. Saukko, K. Lehtoranta et al., "The formation and physical properties of the particle emissions from a natural gas engine," Fuel, vol. 162, pp. 155-161, 2015.

[6] L.-P. Yang, E.-Z. Song, S.-L. Ding, R. J. Brown, N. Marwan, and X.-Z. Ma, "Analysis of the dynamic characteristics of combustion instabilities in a pre-mixed lean-burn natural gas engine," Applied Energy, vol. 183, pp. 746-759, 2016.

[7] B. Yan, H. Wang, Z. Zheng, Y. Qin, and M. Yao, "The effects of LIVC miller cycle on the combustion characteristics and thermal efficiency in a stoichiometric operation natural gas engine with EGR," Applied Thermal Engineering, vol. 122, pp. 439-450, 2017.

[8] G. T. Chala, A. R. A. Aziz, and F. Y. Hagos, "Combined effect of boost pressure and injection timing on the performance and combustion of CNG in a DI spark ignition engine," International Journal of Automotive Technology, vol. 18, no. 1, pp. 85-96, 2017.

[9] D. K. Srivastava and A. K. Agarwal, "Combustion characteristics of a variable compression ratio laser-plasma ignited compressed natural gas engine," Fuel, vol. 214, pp. 322-329, 2018.

[10] F. Yan, L. Xu, and Y. Wang, "Application of hydrogen enriched natural gas in spark ignition IC engines: from fundamental fuel properties to engine performances and emissions," Renewable and Sustainable Energy Reviews, vol. 82, no. 1, pp. 1457-1488, 2018.

[11] G. T. Chala, A. R. A. Aziz, and F. Y. Hagos, "Natural gas engine technologies: challenges and energy sustainability issue," Energies, vol. 11, no. 11, pp. 1-44, 2018.

[12] K. N. Duc, V. N. Duy, L. H. Dinh et al., "Performance and emission characteristics of a port fuel injected, spark ignition engine fuelled by compressed natural gas," Sustainable Energy Technologies and Assessments, vol. 31, pp. 383-389, 2019.

[13] J. Yang and F. Y. Yi, "Adaptive fuel-air ratio control of compressed natural gas engines," in Proceedings of the 38th Chinese Control Conference, pp. 6470-6474, Guangzhou, China, July 2019.

[14] M. Garg and R. V. Ravikrishna, "In-cylinder flow and combustion modeling of a CNG-fuelled stratified charge engine," Applied Thermal Engineering, vol. 149, pp. 425-438, 2019.

[15] J. Yang, T. L. Shen, and X. H. Jiao, "Model-based stochastic optimal air-fuel ratio control with residual gas fraction of spark ignition engines," IEEE Transactions on Control Systems Technology, vol. 22, no. 3, pp. 895-910, 2014.

[16] O. L. V. Costa and M. D. Fragoso, "Stability result for discretetime linear systems with Markovian jumping parameters," Journal of Mathematical Analysis and Applications, vol. 179, no. 1, pp. 154-178, 1993.

[17] J. B. Heywood, Internal Combustion Engine Fundamentals, McGraw-Hill, New York, NY, USA, 1988.

[18] A. Stotsky, B. Egardt, and S. Eriksson, "Variable structure control of engine idle speed with estimation of unmeasurable disturbances," Journal of Dynamic Systems, Measurement, and Control, vol. 122, no. 4, pp. 599-603, 2000.

[19] E. Hendricks and S. C. Sorenson, "Mean value modeling of spark ignition engines," SAE Paper 900616, SAE International, Warrendale, PA, USA, 1990. 